\title{
The efficacy and safety of PD-1/PD-L1 immune checkpoint inhibitors in treating advanced urothelial cancer: a meta-analysis of clinical trials
}

\author{
Fei $\mathrm{Li}^{1,{ }^{*}}$, Yu Wang ${ }^{1, *}$, Kunfeng Xie ${ }^{1}$, Yunze Fang ${ }^{1}$, Yuejun $\mathrm{Du}^{1}$, Lina $\mathrm{Hou}^{2,{ }^{*}, \&}$, Wanlong $\operatorname{Tan}^{1,{ }^{*}}$ \\ ${ }^{1}$ Department of Urology, Nanfang Hospital, Southern Medical University, Guangzhou, Guangdong 510515, PR China \\ ${ }^{2}$ Department of Healthy Management, Nanfang Hospital, Southern Medical University, Guangzhou, Guangdong \\ 510515, PR China \\ ${ }^{*}$ Equal contribution
}

Correspondence to: Lina Hou, Wanlong Tan; email: Inhou2010@126.com, https://orcid.org/0000-0003-3484-9929;

twl@smu.edu.cn

Keywords: urothelial cancer, immunotherapy, efficacy, safety, meta-analysis

Received: December 1, $2020 \quad$ Accepted: May 10, $2021 \quad$ Published: August 23, 2021

Copyright: (C) $2021 \mathrm{Li}$ et al. This is an open access article distributed under the terms of the Creative Commons Attribution License (CC BY 3.0), which permits unrestricted use, distribution, and reproduction in any medium, provided the original author and source are credited.

\begin{abstract}
Survival outcomes in advanced urothelial cancer (UC) are dismal. Over the past years, immunotherapy remains an evolving treatment modality for these patients. This meta-analysis was performed to comprehensively evaluate the efficacy and safety of immune checkpoint inhibitors. For this purpose, 18 clinical trials comprising a total of 3,144 patients were identified from the PubMed database up to September 2020. Overall, the objective response rate (ORR) to PD-1/PD-L1 inhibitors was 0.20 [95\% confidence intervals (Cl) $0.17-0.23$ ]. Furthermore, the pooled 1-year overall survival (OS) and 1-year progression-free survival (PFS) rates were 0.43 (95\% $\mathrm{Cl} 0.33-0.53)$ and $0.19(95 \% \mathrm{Cl} 0.17-0.21)$, respectively. The summary rates of any-grade and grade $\geq 3$ adverse events (AEs) were $0.66(95 \% \mathrm{Cl} 0.58-0.74)$ and $0.13(95 \% \mathrm{Cl} 0.09-0.18)$, respectively. Among the different subgroups, PD-1/PD-L1 inhibitors elicited a promising ORR in patients with lymph node-only metastasis compared to those with visceral metastasis $(0.41 \mathrm{VS}$. 0.17$)$. Additionally, patients with primary tumor in the lower tract had higher ORR compared to those with primary tumor in the upper tract (0.24 VS. 0.15). Briefly speaking, this immunotherapy protocol showed an encouraging efficacy and acceptable safety profile in the treatment of advanced UC. Moreover, our findings provided potential clinical significance for patients with lymph node-only metastasis or primary tumor in the lower tract. However, these exciting findings need further confirmation.
\end{abstract}

\section{INTRODUCTION}

Bladder cancer is the 10th most prevalent form of cancer worldwide. Advanced urothelial cancer (UC) has a poor prognosis, and the efficacy of therapeutic options currently available for these patients is limited $[1,2]$. Nowadays, platinum is still the first-line chemotherapy for advanced UC [3]. Unfortunately, about 30\% of patients with advanced UC are considered to be platinum ineligible because of impaired renal function, comorbidities or other reasons. Thus, developing effective treatment strategies remains quite challenging
[4]. In addition, the clinical efficacy of these second-line drugs including vinflunine or taxanes and gemcitabine in the treatment of advanced UC is not ideal, and there is still an urgent need for another effective treatment [5].

In recent years, immunotherapy has become an increasingly promising therapeutic method for advanced $\mathrm{UC}$, with immune checkpoint inhibitors being able to halt immune evasion of cancer cells by preventing programmed cell death protein 1 (PD-1) from binding to its ligand [6]. In the past few years, the U.S. Food and Drug Administration (FDA) have approved 6 immune 
checkpoint inhibitors (Atezolizumab, Pembrolizumab, Durvalumab, Nivolumab, Avelumab and Tislelizumab) for clinical treatment of patients diagnosed with advanced UC or cisplatin-ineligible, who were previously treated with first-line standard chemotherapy [7].

The efficacy and safety profile of PD-1/PD-L1 inhibitors are the major concern related to immunotherapy. Recently, a meta-analysis conducted by Zhang et al., including studies performed before July 2019, reported that the pooled ORR of immune checkpoint inhibitors was 0.20 , and the 1-year OS and 1-year PFS rates were 0.50 and 0.17 , respectively. The summary frequencies of any-grade and grade $\geq 3$ AEs were 0.65 and 0.11 , respectively [8]. However, 6 other studies on the association between immune checkpoint inhibitors and advanced UC were carried out last year. Thus, we systematically collected available published data and performed an updated meta-analysis to investigate the efficacy and safety of PD-1/PD-L1 inhibitors in the treatment of advanced UC patients. The outcomes were then compared across subgroups stratified by different PD-L1 expression levels, studied drugs, and metastasis or primary tumor locations.

\section{MATERIALS AND METHODS}

\section{Literature search}

We conducted a thorough search of the PubMed database to identify the relevant literature until October 2020, using the following research terms: "metastatic bladder cancer" OR "metastatic urothelial carcinoma" OR "bladder cancer" OR "transitional cell carcinoma" AND "PD-L1" OR "PD-1" OR "immunotherapy" OR "immune checkpoint inhibitor" OR "Pembrolizumab" OR "Atezolizumab" OR "Avelumab" OR "Durvalumab" OR "Tislelizumab" OR "Nivolumab" [8]. The search was focused on human studies, without restriction on language. We also checked for relevant articles and their references to search all eligible literature. Two authors (Y.W. and K.F.X.) independently screened the literature for eligibility and any disagreements were resolved by reaching a consensus.

\section{Inclusion and excluded criteria}

Our meta-analysis included studies which met the following criteria: (1) Patients in all studies were exclusively diagnosed with advanced UC. (2) Patients were treated with PD-1/PD-L1 inhibitors including Atezolizumab, Avelumab Durvalumab, Nivolumab, Pembrolizumab, and Tislelizumab. (3) Studies were all clinical trials assessing PD-1/PD-L1 inhibitors. (4) Studies reported the data on efficacy and safety of PD-1/ PD-L1 inhibitors, including following indexes: ORR, 1-year PFS rate, 1-year OS rate, rates of any-grade and grade $\geq 3$ AEs.

The exclusion criteria were as follows: (1) Duplicates; (2) Lack of required data (3) case reports, reviews, ecological analyses and off-topic studies, etc. Besides, if multiple studies were conducted from the same or overlapping cohort, only the most informative one was included.

\section{Data extraction}

Two authors (Y.W. and Y.Z.F.) independently extracted data from the selected studies using a standardized data collection form. Any discrepancy was resolved by discussing and reaching a consensus. The extracted information was: the name of first authors, the publication year, phase of research, use of drugs in the trial, median follow-up time, PD-1/PD-L1 inhibitors used as the first line or the second line, the control group of each clinic trail, dosage of drugs, number of recipients, age of participants, ORR, 1-year PFS rate, 1 -year OS rate, rates of any-grade and grade $\geq 3$ AEs.

\section{Outcomes and quality assessment}

The outcome measures included the ORR, 1-year OS rate, 1-year PFS rate, rates of any-grade and grade $\geq 3$ AEs. Quality assessment of the studies was conducted independently by two authors (F.L and Y.J.D) based on the Jadad score by RevMan 5.3 [9], and diverging opinions were resolved by discussion.

\section{Statistical analysis}

In this meta-analysis, we presented evaluation indicators with percentages and its 95\% confidence intervals (95\% CI). Both the fixed- and random-effects methods were used to estimate the overall association. Statistical heterogeneity among the included studies was measured by the Q-statistic (Statistical significance was set at $P<0.05)$ and $I^{2}$ statistic $[10,11]$. We calculated the pooled ORR, 1-year PFS rate and 1-year OS rate with $95 \%$ CI to evaluate the efficacy profile of PD-1/ PD-L1 inhibitors [8]. Similarly, we computed the overall rates for any-grade and grade $\geq 3$ AEs to evaluate the safety of immune check point inhibitors.

Subgroup analyses were conducted to measure possible sources of heterogeneity on the basis of different PD-L1 expression levels, PD-L1/PD-1 inhibitors, studied drugs, visceral or lymph node-only metastasis, and primary tumor in the upper or lower tract. Sensitivity analyses were designed to evaluate the robustness of the results. In addition, Egger's test and Begg's were utilized to assess for potential bias [12]. All statistical 
analyses were performed using RevMan 5.3 (Cochrane Collaboration, Oxford, UK) and the "meta" package in the R software 3.6.0 (R Foundation for Statistical Computing, Vienna, Austria). A two-tailed $P$ value $<0.05$ was considered statistically significant.

\section{RESULTS}

\section{Literature search results}

A flow chart of our selection process was illustrated in Figure 1. A total of 1,409 articles were identified after our search. Of those, 33 were considered to be preliminary selected articles for further review after excluding duplicate articles and screening the titles and abstracts to determine their relevance. After a full-text review of the remaining 33 articles, 7 articles were excluded due to the fact that they did not report relevant outcomes. Meanwhile the remaining 8 articles were excluded since their participant cohorts overlapped with other studies. Finally, we included a total of 18 articles in our meta-analysis [12-29] (Figure 1).

\section{Characteristics of included studies}

The characteristics of the included studies were shown in Table 1. The enrolled studies were published between 2014 and 2020. The Jadad score of each included study ranged from 3 to 5 , no study received a low-quality Jadad score, validating our selection criteria. Moreover, 11 studies assessed PD-L1 inhibitors (Atezolizumab $=7$ [13-19], Durvalumab $=2$ $[20,21]$, Avelumab $=2[22,23]$, whereas 7 articles studied PD-1 inhibitors (Pembrolizumab $=4$ [24-27], Nivolumab $=2[28,29]$, Tislelizumab $=1$ [30]). Our meta-analysis involved 3,144 patients diagnosed with advanced UC and the medium follow-up time of included studies ranged from 2.3 to 37.8 months (Table 1).

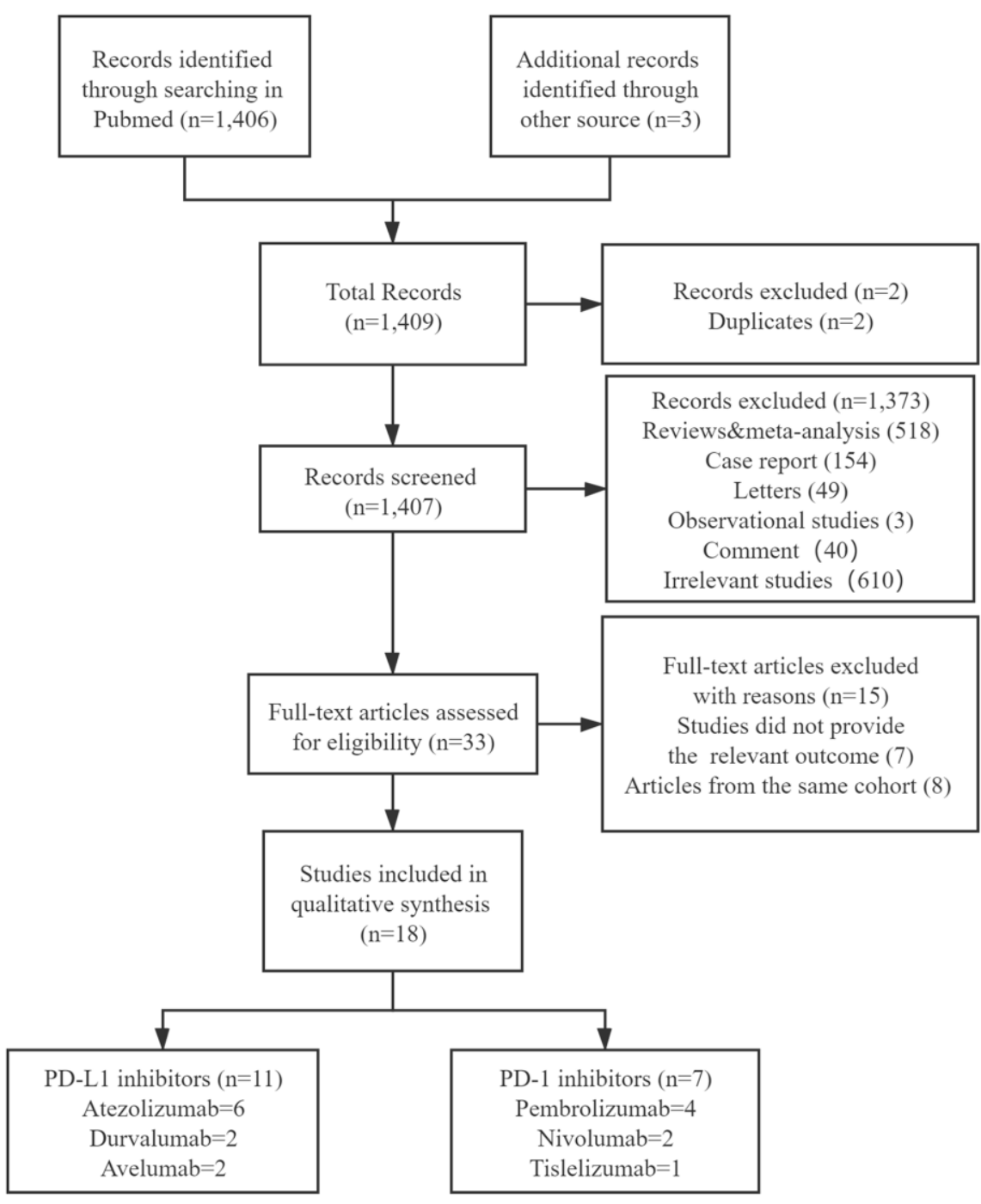

Figure 1. Flow chart of the study selection procedure. 
Table 1. Baseline characteristics and data of the included studies using PD-1/PD-L1 inhibitors.

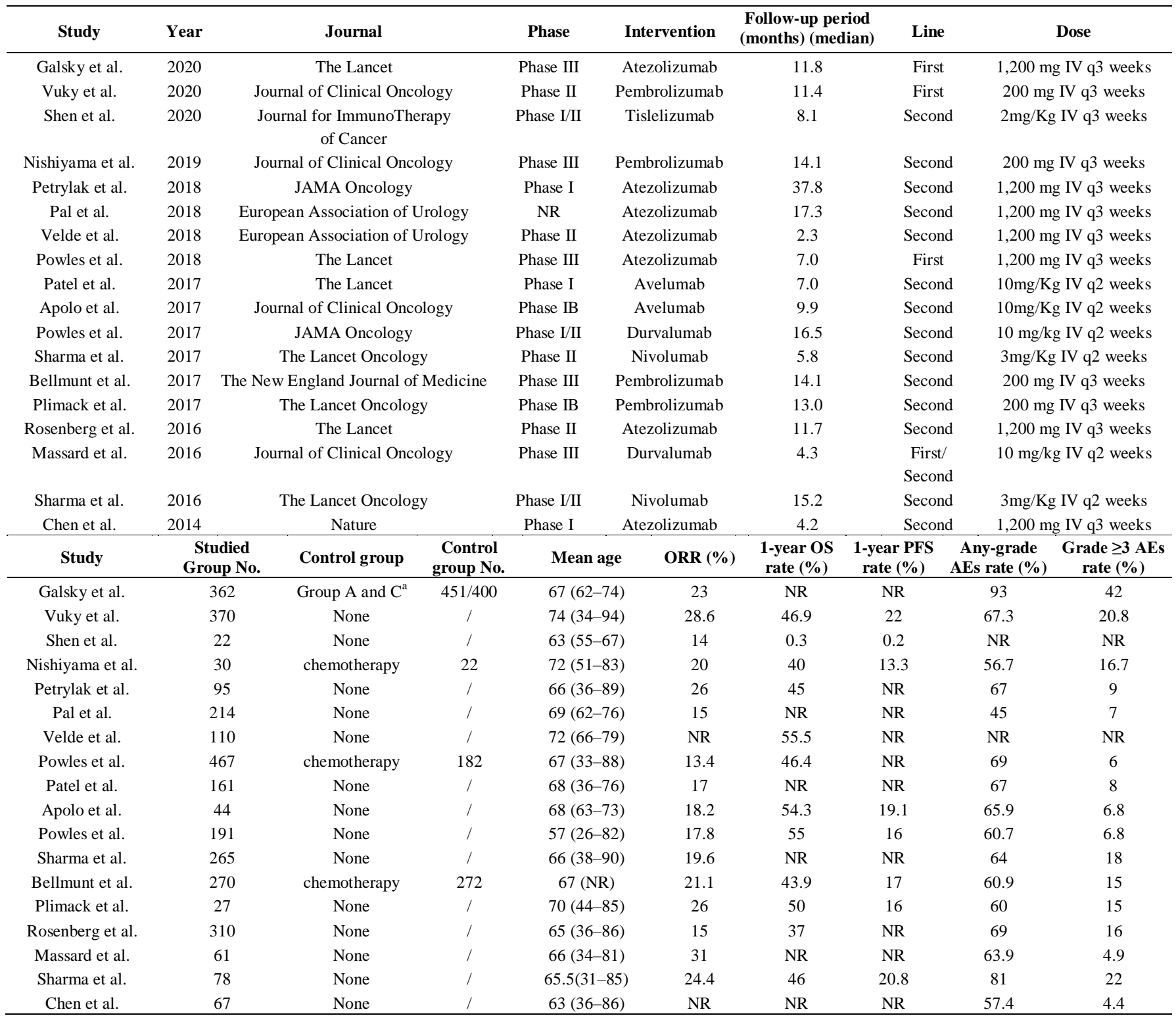

${ }^{a}$ Group A (Atezolizumab plus chemotherapy) and group C (placebo plus chemotherapy).

Abbreviations: AE: adverse event; IV: intravenous; NR: not reported; ORR: objective response rate; OS: overall survival; PFS: progression-free survival.

\section{Efficacy assessment}

We used the pooled ORR, 1-year PFS rate and 1-year OS rate to evaluate the efficacy of PD-1/PD-L1 immune checkpoint inhibitors in treatment of advanced UC. Figure 2 delineates the ORR for advanced UC using the random-effects model based on 16 studies [12-16, 1929] with a sample size of 2,843 individuals. The pooled ORR was 0.20 (95\% CI 0.17-0.23, Figure 2A). The pooled complete response $(\mathrm{CR})$ and partial response (PR) were 0.05 and 0.14 , respectively. Furthermore, 12 studies $[12,13,15,17,22-29]$ were assessed for the 1year OS rate, with the pooled 1-year OS rate being 0.43 (95\% CI 0.33-0.53, Figure 2B). Additionally, 8 studies [22-29] were assessed for the 1-year PFS rate, and the pooled outcome was 0.19 (95\% CI 0.17-0.21, Figure 2C). Substantial heterogeneity was observed across studies in terms of ORR $\left(I^{2}=68.7 \%, p<0.0001\right.$, Figure $2 \mathrm{~A})$ and 1 -year OS rate $\left(I^{2}=95.1 \%, p<0.0001\right.$, Figure 2B), but no indication of heterogeneity was shown in terms of the 1 -year PFS rate $\left(I^{2}=0 \%, p=0.639\right.$, Figure 2C) (Table 2).

We used Begg's and Egger's tests to conduct asymmetry tests and measure the publication bias. The Begg's test did not establish evidence of publication bias after analysis of the ORR $(P=$ $0.195), 1$-year OS rate $(P=0.593)$ and 1 -year PFS rate $(P=0.298)$. Likewise, the Egger's test did not point out evidence of publication bias with respect to 
the ORR $(P=0.653), 1$-year OS rate $(P=0.493)$, and 1-year PFS $(P=0.266)$.

When studies were stratified based on different PD-L1 expression levels, PD-L1 or PD-1 inhibitors, studied drugs, visceral or lymph node-only metastasis and in the upper or lower tract 9 [12-16, 19-22] studies reported the efficacy of PD-L1 inhibitors, with the pooled ORR being 0.18 (95\% CI $0.15-0.21, I^{2}=63.3 \%, P=0.005$ ). Whilst 7 studies [23-29] reported the efficacy of PD-1 inhibitors, and the pooled ORR was 0.23 (95\% CI $0.21-$ $\left.0.26, I^{2}=42 \%, P=0.114\right)$. PD-1 inhibitors had a better

\section{A}

Study

Galsky 2020 Atezolizumab [16] Vuky 2020 Pembrolizumab [23] Shen 2020 Tislelizumab [29] Nishiyama 2019 Pembrolizumab [25] Petrylak 2018 Atezolizumab [12] Pal 2018 Atezolizumab [14]

Powles 2018 Atezolizumab [15] Patel 2017 Avelumab [21] Apolo 2017 Avelumab [22] Powles 2017 Durvalumab [19] Sharma 2017 Nivolumab [27] Bellmunt 2017 Pembrolizumab [24] Plimack 2017 Pembrolizumab [26] Rosenberg 2016 Atezolizumab [13] Massard 2016 Durvalumab [20] Sharma 2016 Nivolumab [28]

Fixed effect model

Random effects model

Heterogeneity: $I^{2}=69 \%, \tau^{2}=0.0020, p<0.01$
Events Total

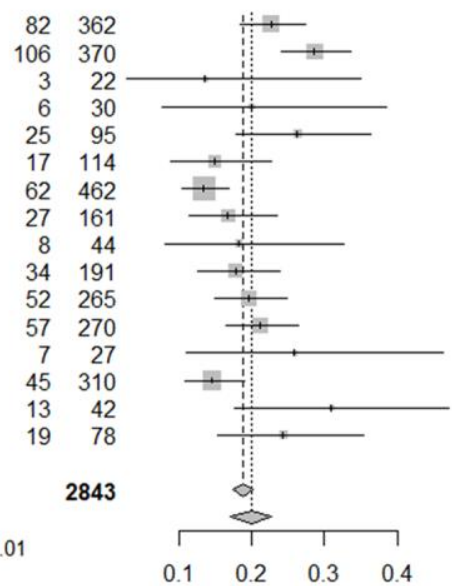

Proportion $\quad 95 \%-\mathrm{Cl} \quad \begin{aligned} & \text { Weight } \\ & \text { (fixed) (random) }\end{aligned}$

0.23 [0.18:0.27] $10.9 \%$

$0.14[0.03 ; 0.35] \quad 1.0 \% \quad 2.9 \%$

$0.20[0.08 ; 0.39] \quad 1.0 \% \quad 2.9 \%$

$0.26[0.18 ; 0.36] \quad 2.6 \% \quad 5.3 \%$

$0.15[0.09 ; 0.23] \quad 4.7 \% \quad 6.9 \%$

$0.13[0.10 ; 0.17] \quad 21.0 \% \quad 9.6 \%$

$0.17[0.11 ; 0.23] \quad 6.1 \% \quad 7.5 \%$

$0.18[0.08 ; 0.33] \quad 1.6 \% \quad 4.0 \%$

$0.18[0.13 ; 0.24] \quad 6.9 \% \quad 7.8 \%$

$0.20[0.15 ; 0.25] \quad 8.9 \% \quad 8.3 \%$

$0.21[0.16 ; 0.26] \quad 8.6 \% \quad 8.3 \%$

$0.26[0.11 ; 0.46] \quad 0.7 \% \quad 2.3 \%$

$0.15[0.11 ; 0.19] \quad 13.2 \% \quad 9.0 \%$

$0.31[0.18 ; 0.47] \quad 1.0 \%-3.0 \%$

$0.24[0.15 ; 0.35] \quad 2.2 \% \quad 4.9 \%$

$0.19[0.17 ; 0.20] 100.0 \%$ $0.20[0.17 ; 0.23] \quad-\quad 100.0 \%$
$0.29[0.24 ; 0.34] \quad 9.6 \% \quad 8.5 \%$

\section{B}

Study

Vuky 2020 Pembrolizumab [23] Shen 2020 Tislelizumab [29] Nishiyama 2019 Pembrolizumab [25]

Petrylak 2018 Atezolizumab [12] Velde 2018 Atezolizumab [17] Powles 2018 Atezolizumab [15]

Powles 2017 Durvalumab [27] Bellmunt 2017 Pembrolizumab [24] Plimack 2017 Pembrolizumab [26] Rosenberg 2016 Atezolizumab [13] Sharma 2016 Nivolumab [28]

Fixed effect model Random effects model Heterogeneity: $I^{2}=95 \%, \tau^{2}=0.0268, p<0.01$

Events Total

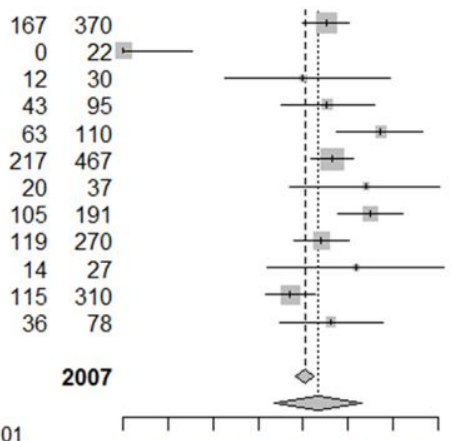

$\begin{array}{llllllll}0 & 0.1 & 0.2 & 0.3 & 0.4 & 0.5 & 0.6 & 0.7\end{array}$ Apolo 2017 Avelumab [22]

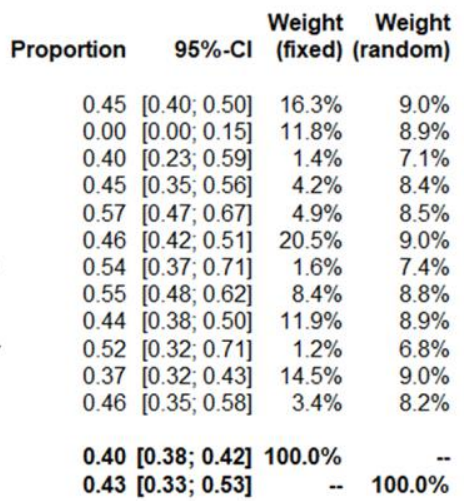

$0.43[0.33 ; 0.53] \quad-\quad 100.0 \%$

\section{C} Study

Vuky 2020 Pembrolizumab [23] Shen 2020 Tislelizumab [29] Nishiyama 2019 Pembrolizumab [25] Apolo 2017 Avelumab [22] Powles 2017 Durvalumab [27] Bellmunt 2017 Pembrolizumab [24] Plimack 2017 Pembrolizumab [26] Sharma 2016 Nivolumab [28]

Fixed effect model Random effects model Heterogeneity: $I^{2}=0 \%, \tau^{2}=0, p=0.64$

\section{Events Total}

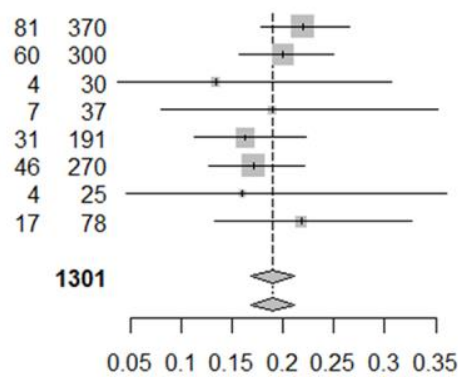

Weight Weight Proportion 95\%-Cl (fixed) (random)

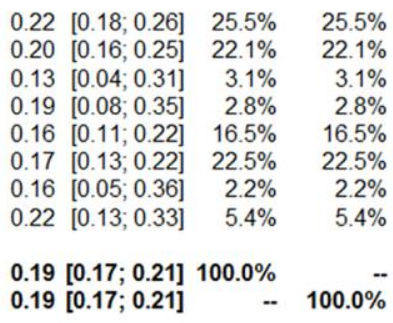

Figure 2. Forest plot of the efficacy of immune checkpoint inhibitors in treating patients with advanced urothelial cancer. (A) Pooled objective response rate. (B) Pooled 1-year overall survival rate. (C) Pooled 1-year progress free survival rate. The diamonds represent the pooled indexes. The line crossing the square represents the $95 \% \mathrm{Cl} . I^{2}$ indicates the heterogeneity in each subgroup metaanalysis. $P$ demonstrates the significance of differences between the subgroups. 
Table 2. The outcomes of the efficacy of PD-1/PD-L1 inhibitors.

\begin{tabular}{|c|c|c|c|c|c|c|c|}
\hline Analysis Specifications & Studies & Total event & Total population & $\begin{array}{c}\text { Proportion } \\
(\mathbf{9 5 \%} \text { CI) }\end{array}$ & $\begin{array}{c}P \text { Value } \\
\text { Heterogeneity }\end{array}$ & $\begin{array}{c}P \\
\text { Egger's } \\
\end{array}$ & $\begin{array}{c}P \\
\text { Begg's }\end{array}$ \\
\hline 1-year OS rate & 12 & 911 & 2007 & $0.43(0.33-0.53)$ & $<0.0001$ & 0.493 & 0.593 \\
\hline 1-year PFS rate & 8 & 250 & 1301 & $0.19(0.17-0.21)$ & 0.639 & 0.266 & 0.298 \\
\hline ORR & 16 & 563 & 2843 & $0.20(0.17-0.23)$ & $<0.0001$ & 0.653 & 0.195 \\
\hline $\mathrm{CR}$ & 14 & 136 & 2531 & $0.05(0.04-0.06)$ & 0.005 & 0.020 & 0.502 \\
\hline PR & 14 & 357 & 2531 & $0.14(0.11-0.16)$ & 0.003 & 0.556 & 1.000 \\
\hline SD & 14 & 548 & 2531 & $0.21(0.19-0.24)$ & 0.002 & 0.825 & 0.584 \\
\hline PD & 14 & 1078 & 2531 & $0.42(0.35-0.48)$ & $<0.0001$ & 0.829 & 0.661 \\
\hline \multicolumn{8}{|l|}{ Drug of study } \\
\hline PD-L1inhibitor & 9 & 313 & 1781 & $0.18(0.15-0.21)$ & 0.009 & 0.086 & 0.251 \\
\hline Atezolizumab & 5 & 231 & 1343 & $0.18(0.14-0.22)$ & 0.004 & 0.289 & 0.221 \\
\hline Avelumab & 2 & 35 & 205 & $0.17(0.12-0.22)$ & 0.830 & - & - \\
\hline Durvalumab & 2 & 47 & 233 & $0.20(0.14-0.25)$ & 0.060 & - & - \\
\hline PD-1 inhibitor & 7 & 250 & 1062 & $0.23(0.21-0.26)$ & 0.114 & 0.560 & 1.000 \\
\hline Nivolumab & 2 & 71 & 343 & $0.21(0.16-0.25)$ & 0.384 & - & - \\
\hline Pembrolizumab & 4 & 176 & 697 & $0.25(0.22-0.28)$ & 0.149 & 0.806 & 1.000 \\
\hline \multicolumn{8}{|l|}{ Expression of PD-L1 } \\
\hline PD-L1 (+) & 7 & 134 & 503 & $0.26(0.22-0.29)$ & 0.026 & 0.093 & 0.133 \\
\hline PD-L1 (-) & 7 & 63 & 468 & $0.12(0.06-0.17)$ & 0.001 & 0.162 & 1.000 \\
\hline \multicolumn{8}{|l|}{ Location of metastasis } \\
\hline Visceral & 7 & 178 & 1013 & $0.17(0.12-0.23)$ & $<0.0001$ & 0.337 & 0.548 \\
\hline Lymph node-only & 7 & 70 & 169 & $0.41(0.32-0.50)$ & 0.265 & 0.723 & 0.649 \\
\hline \multicolumn{8}{|l|}{ Location of primary tumor } \\
\hline Upper-tract & 2 & 16 & 105 & $0.15(0.08-0.21)$ & 0.366 & - & - \\
\hline Lower-tract & 2 & 111 & 425 & $0.24(0.13-0.35)$ & 0.012 & - & - \\
\hline
\end{tabular}

Abbreviations: ORR: objective response rate; OS: overall survival; PFS: progression-free survival; CR: complete response; PR: partial response; SD: stable disease; PD: progressive disease.

efficacy profile compared with PD-L1 inhibitors. In fact, Pembrolizumab had a higher ORR $(0.25,95 \%$ CI $\left.0.22-0.28, I^{2}=44 \%, P=0.149\right)$ than all the reported immune checkpoint inhibitors. The pooled ORRs of drug subgroups for Atezolizumab, Durvalumab, Nivolumab and Avelumab were 0.18 (95\% CI 0.14$\left.0.22, I^{2}=77.2 \%, P=0.002\right), 0.20$ (95\% CI 0.14-0.25, $\left.I^{2}=33 \%, P=0.004\right), 0.21\left(95 \%\right.$ CI $0.16-0.25, I^{2}=0 \%$, $P=0.384)$ and $0.17(95 \%$ CI $0.12-0.22, I=0 \%, P=$ 0.830 ), respectively (Table 2 ).

The PD-L1 expression levels of included patient tumor samples were evaluated by immunohistochemistry. Subsequently, the group with PD-L1 expression $\geq 1 \%$ was denoted as the positive group, and the group with PD-L1 expression $<1 \%$ as the negative group. The pooled ORR of the $\mathrm{PD}-\mathrm{L} 1$-positive group $(\mathrm{ORR}=$ $0.26,95 \%$ CI $0.22-0.29, I^{2}=58 \%, P=0.026$ ) indicated a better efficacy than the PD-L1-negative group $\left(\mathrm{ORR}=0.12,95 \%\right.$ CI $0.06-0.17, I^{2}=74 \%, P=$ 0.001) (Table 2). Furthermore, patients with lymph node-only metastasis (ORR $=0.41,95 \%$ CI $0.34-0.48$, $\left.I^{2}=22 \%, P=0.265\right)$ experienced a better efficacy results than those with visceral metastasis $(\mathrm{ORR}=$
$0.17,95 \%$ CI $0.12-0.23, I^{2}=79 \%, P<0.0001$ ) (Figure $3 \mathrm{~A}$ and $3 \mathrm{~B}$ ). Moreover, the overall ORR for studies with the primary tumor located in the lower tract was 0.24 (95\% CI $0.12-0.23)$, with variability detected ( $p$-value for heterogeneity $\left.=0.012, I^{2}=84 \%\right)$. There was less evidence of heterogeneity in studies with the primary tumor located in the upper tract $(\mathrm{ORR}=0.15$, $95 \%$ CI $0.08-0.21, p$-value for heterogeneity $=0.366$, $I^{2}=0 \%$ ) (Figure 3C and 3D).

\section{Safety assessment}

The rates of any-grade and grade $\geq 3$ AEs were used to gauge the safety of PD-1/PD-L1 inhibitors in the treatment of metastatic UC. The pooled rates of anygrade and grade $\geq 3$ AEs rates are presented in Figure 4 . The summary outcomes for any-grade and grade $\geq 3$ AEs were 0.66 (95\% CI 0.58-0.74) and 0.13 (95\% CI 0.09-0.18), respectively. Obvious heterogeneity was found in the pooled estimation of the rate of any-grade AEs $\left(I^{2}=95.7 \%, P<0.0001\right)$ and grade $\geq 3$ AEs $\left(I^{2}=\right.$ 93.5\%, $P<0.0001)$. Thence, subgroup analysis based on PD-L1/PD-1 inhibitors was performed to explore the sources of heterogeneity. The significant evidence of 
publication bias was not indicated by the Egger's and Begg's tests.

Overall, the pooled rate of any-grade AEs in the PD-1 group $\left(0.66,95 \%\right.$ CI $0.60-0.72 I^{2}=69 \%, P=$ $0.007)$ was similar to that in the PD-L1 group (0.66, $95 \%$ CI $\left.0.55-0.77, I^{2}=97 \%, P<0.0001\right)$.
Interestingly, the summary rate of grade $\geq 3$ AEs in the PD-1 group $\left(0.18,95 \% \mathrm{CI}, 0.16-0.20, I^{2}=0 \%\right.$, $P=0.478)$ was significantly higher than the PD-L1 group's $\left(0.11,95 \%\right.$ CI $0.05-0.17 I^{2}=95.1 \%, P<$ 0.0001) (Table 3). Thus, PD-1/PD-L1 immune checkpoint inhibitors have an acceptable safety outcome.

A

\section{Study}

Vuky 2020 Pembrolizumab [23]

Patel 2017 Avelumab [21]

Apolo 2017 Avelumab [22]

Powles 2017 Durvalumab [19]

Sharma 2017 Nivolumab [27]

Rosenberg 2016 Atezolizumab [13]

Massard 2016 Durvalumab [20]

Fixed effect mode

Random effects model

Heterogeneity: $I^{2}=79 \%, \tau^{2}=0.0037, p<0.01$

\section{Events Total}

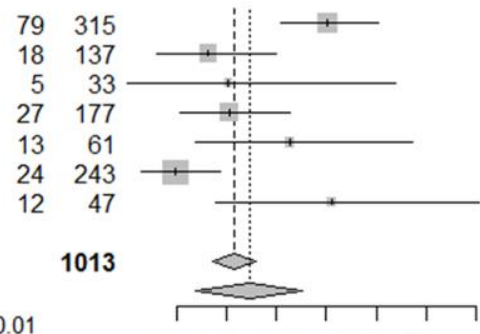

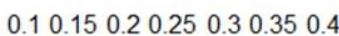

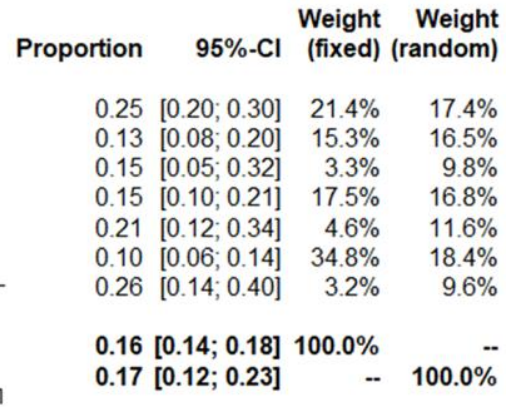

$0.17[0.12 ; 0.23] \quad--100.0 \%$

\section{B}

Study

Vuky 2020 Pembrolizumab [23]

Patel 2017 Avelumab [21]

Apolo 2017 Avelumab [22]

Powles 2017 Durvalumab [19]

Sharma 2017 Nivolumab [27]

Rosenberg 2016 Atezolizumab [13]

Massard 2016 Durvalumab [20]

Fixed effect model

Random effects model

Heterogeneity: $I^{2}=22 \%, \tau^{2}=0.0028, p=0.27$

Events Total

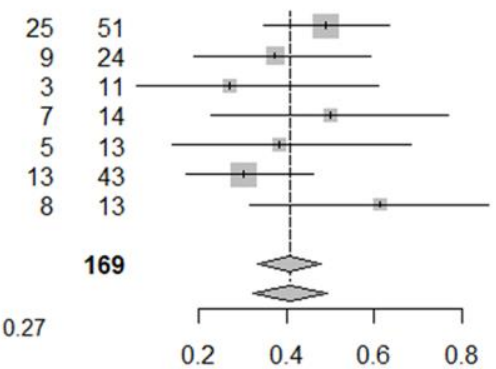

Proportion $\quad 95 \%-\mathrm{Cl} \quad \begin{gathered}\text { Weight } \\ \text { (fixed) (random) }\end{gathered}$

$0.49[0.35 ; 0.63] \quad 27.9 \% \quad 24.5 \%$

$0.38[0.19 ; 0.59] \quad 14.0 \% \quad 15.0 \%$

$0.27[0.06 ; 0.61] \quad 7.6 \% \quad 9.0 \%$

$0.50[0.23 ; 0.77] \quad 7.7 \% \quad 9.1 \%$

$0.38[0.14 ; 0.68] \quad 7.5 \% \quad 9.0 \%$

$0.30[0.17 ; 0.46] \quad 27.9 \% \quad 24.4 \%$

$0.62[0.32 ; 0.86] \quad 7.5 \% \quad 9.0 \%$

$0.41[0.34 ; 0.48] 100.0 \% \quad 100 \%$

\section{C}

Study

Patel 2017 Avelumab [21] Vuky 2020 Pembrolizumab [23]

Fixed effect model

Random effects model

Heterogeneity: $I^{2}=0 \%, \tau^{2}=0, p=0.37$
Events Total

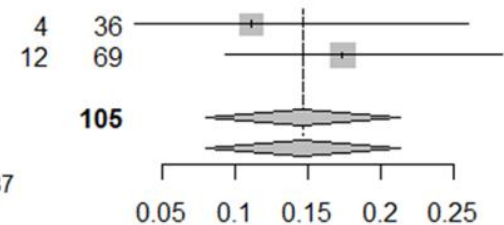

Proportion Weight Weight

$0.11[0.03 ; 0.26] \quad 43.1 \% \quad 43.1 \%$

$0.17[0.09 ; 0.28] \quad 56.9 \% \quad 56.9 \%$

$0.15[0.08 ; 0.21] 100.0 \% \quad-$
D

Study

Patel 2017 Avelumab [21] Vuky 2020 Pembrolizumab [23]

Fixed effect model

Random effects model

Heterogeneity: $l^{2}=84 \%, \tau^{2}=0.0050, p=0.01$
Events Total

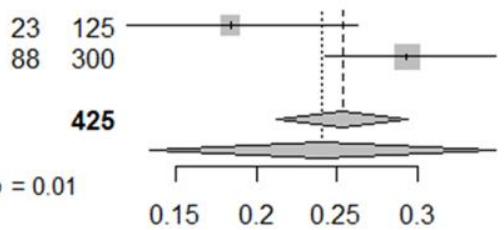

Proportion $95 \%-\mathrm{Cl} \quad \begin{gathered}\text { Weight } \\ \text { (fixed) (random) }\end{gathered}$

$0.18[0.12 ; 0.26] \quad 36.5 \% \quad 47.9 \%$ $0.29[0.24 ; 0.35] \quad 63.5 \% \quad 52.1 \%$

$0.25[0.21 ; 0.29] 100.0 \%$ $0.24[0.13 ; 0.35] \quad-\quad 100.0 \%$

Figure 3. Forest plot of the subgroup outcomes. (A) Pooled ORR of patients with visceral metastasis. (B) Pooled ORR of patients with lymph node only metastasis. (C) Pooled ORR of patients with the primary tumor in the upper tract. (D) Pooled ORR of patients with the primary tumor in the lower tract. The diamonds represent the pooled indexes. The line crossing the square represents the $95 \% \mathrm{Cl}$. $I^{2}$ indicates the heterogeneity in each subgroup meta-analysis. $P$ demonstrates the significance of differences between the subgroups. 


\section{DISCUSSION}

Advanced UC patients have a poor prognosis. Currently, platinum-based drugs are the therapeutic mainstay for these patients and there has been a lack of effective second-line drugs [31]. Patients with advanced UC still have a lack of effective treatment regimens to slow the disease's progression long enough for the development of immunotherapy strategy [32, 33]. In recent years, PD-1/PD-L1 immune checkpoint inhibitors remains an evolving treatment modality for advanced UC [34]. To date, FDA has approved 6 immune checkpoint inhibitors for the treatment of advanced UC patients who were previously treated with standard chemotherapy and for those ineligible to the standard chemotherapy.
A Study

Galsky 2020 Atezolizumab [16] Vuky 2020 Pembrolizumab [23] Nishiyama 2019 Pembrolizumab [25] Petrylak 2018 Atezolizumab [12] Pal 2018 Atezolizumab [14] Powles 2018 Atezolizumab [15] Patel 2017 Avelumab [21] Apolo 2017 Avelumab [22] Powles 2017 Durvalumab [19] Sharma 2017 Nivolumab [27] Bellmunt 2017 Pembrolizumab [24] Plimack 2017 Pembrolizumab [26] Rosenberg 2016 Atezolizumab [13] Massard 2016 Durvalumab [20] Sharma 2016 Nivolumab [28] Chen 2014 Atezolizumab [18]

Fixed effect model Random effects model Heterogeneity: $I^{2}=96 \%, \tau^{2}=0.0221, p<0.01$

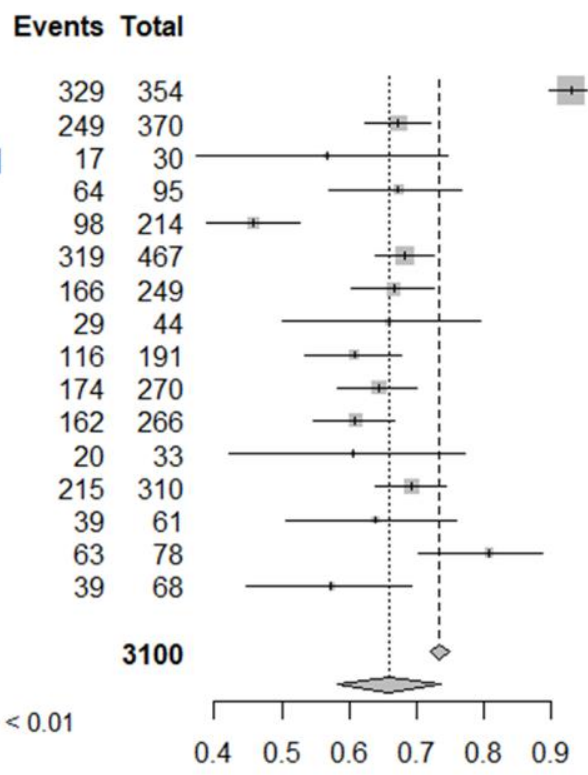

Weight Weight Proportion $\quad 95 \%-\mathrm{Cl}$ (fixed) (random)

$0.93[0.90 ; 0.95] \quad 30.5 \% \quad 6.8 \%$ $0.67[0.62 ; 0.72] \quad 9.5 \% \quad 6.7 \%$ $0.57[0.37 ; 0.75] \quad 0.7 \% \quad 5.0 \%$ $0.67[0.57 ; 0.77] \quad 2.4 \% \quad 6.2 \%$ $0.46[0.39 ; 0.53] \quad 4.9 \% \quad 6.5 \%$ $0.68[0.64 ; 0.73] \quad 12.2 \% \quad 6.7 \%$ $0.67[0.60 ; 0.72] \quad 6.3 \% \quad 6.6 \%$ $0.66[0.50 ; 0.80] \quad 1.1 \% \quad 5.6 \%$ $0.61[0.53 ; 0.68] \quad 4.5 \% \quad 6.5 \%$ $0.64[0.58 ; 0.70] \quad 6.7 \% \quad 6.6 \%$ $0.61[0.55 ; 0.67] \quad 6.3 \% \quad 6.6 \%$ $0.61[0.42 ; 0.77] \quad 0.8 \% \quad 5.2 \%$ $0.69[0.64 ; 0.74] \quad 8.2 \% \quad 6.7 \%$ $0.64[0.51 ; 0.76] \quad 1.5 \% \quad 5.9 \%$ $0.81[0.70 ; 0.89] \quad 2.8 \% \quad 6.3 \%$ $0.57[0.45 ; 0.69] \quad 1.6 \% \quad 5.9 \%$

$0.73[0.72 ; 0.75] 100.0 \% \quad--$ $0.66[0.58 ; 0.74] \quad-\quad 100.0 \%$
B Study

Galsky 2020 Atezolizumab [16] Vuky 2020 Pembrolizumab [23] Nishiyama 2019 Pembrolizumab [25] Petrylak 2018 Atezolizumab [12] Pal 2018 Atezolizumab [14] Powles 2018 Atezolizumab [15] Patel 2017 Avelumab [21] Apolo 2017 Avelumab [22] Powles 2017 Durvalumab [19] Sharma 2017 Nivolumab [27] Bellmunt 2017 Pembrolizumab [24] Plimack 2017 Pembrolizumab [26] Rosenberg 2016 Atezolizumab [13] Massard 2016 Durvalumab [20] Sharma 2016 Nivolumab [28] Chen 2014 Atezolizumab [18]

Fixed effect model

Random effects model Heterogeneity: $I^{2}=93 \%, \tau^{2}=0.0073, p<0.01$

\section{Events Total}

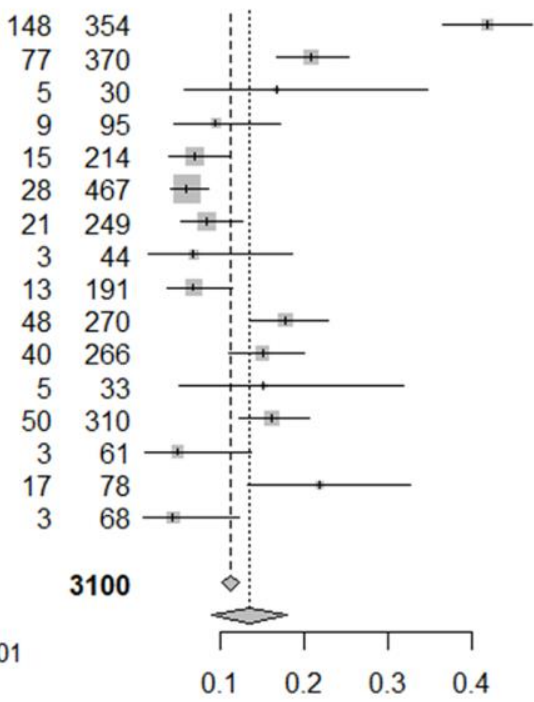

Weight Weight Proportion $\quad 95 \%-\mathrm{Cl} \quad$ (fixed) (random)

\begin{tabular}{|c|c|c|c|}
\hline 0.42 & {$[0.37 ; 0.47]$} & $4.4 \%$ & $6.5 \%$ \\
\hline 0.21 & {$[0.17 ; 0.25]$} & $6.7 \%$ & $6.7 \%$ \\
\hline 0.17 & {$[0.06 ; 0.35]$} & $0.6 \%$ & $4.3 \%$ \\
\hline 0.09 & {$[0.04 ; 0.17]$} & $3.3 \%$ & $6.3 \%$ \\
\hline 0.07 & {$[0.04 ; 0.11]$} & $9.8 \%$ & $6.8 \%$ \\
\hline 0.06 & {$[0.04 ; 0.09]$} & $24.8 \%$ & $7.0 \%$ \\
\hline 0.08 & {$[0.05 ; 0.13]$} & $9.7 \%$ & $6.8 \%$ \\
\hline 0.07 & {$[0.01 ; 0.19]$} & $2.1 \%$ & $5.9 \%$ \\
\hline 0.07 & {$[0.04 ; 0.11]$} & $9.0 \%$ & $6.8 \%$ \\
\hline 0.18 & {$[0.13 ; 0.23]$} & $5.5 \%$ & $6.6 \%$ \\
\hline 0.15 & {$[0.11 ; 0.20]$} & $6.2 \%$ & $6.6 \%$ \\
\hline 0.15 & {$[0.05 ; 0.32]$} & $0.8 \%$ & $4.6 \%$ \\
\hline 0.16 & {$[0.12 ; 0.21]$} & $6.9 \%$ & $6.7 \%$ \\
\hline 0.05 & {$[0.01 ; 0.14]$} & $3.9 \%$ & $6.4 \%$ \\
\hline 0.22 & {$[0.13 ; 0.33]$} & $1.4 \%$ & $5.5 \%$ \\
\hline 0.04 & {$[0.01 ; 0.12]$} & $4.8 \%$ & $6.5 \%$ \\
\hline
\end{tabular}

Figure 4. Forest plot of the safety of immune checkpoint inhibitors in treating patients with advanced urothelial cancer. (A) Pooled any-grade adverse events rate. (B) Pooled grade $\geq 3$ adverse events rate. The diamonds represent the pooled indexes. The line crossing the square represents the $95 \% \mathrm{Cl}$. $I^{2}$ indicates the heterogeneity in each subgroup meta-analysis. $P$ demonstrates the significance of differences between the subgroups. 
Table 3. The outcomes of the any-grade and $\geq 3$ grade AEs rates of PD-1/PD-L1 inhibitors.

\begin{tabular}{cccccccc}
\hline $\begin{array}{c}\text { Analysis } \\
\text { Specifications }\end{array}$ & Studies & $\begin{array}{c}\text { Total } \\
\text { event }\end{array}$ & $\begin{array}{c}\text { Total } \\
\text { population }\end{array}$ & Proportion $(\mathbf{9 5 \%}$ CI) & $\begin{array}{c}\boldsymbol{P} \text { Value } \\
\text { Heterogeneity }\end{array}$ & $\begin{array}{c}\boldsymbol{P} \\
\text { Egger's }\end{array}$ & $\begin{array}{c}\boldsymbol{P} \\
\text { Begg's }\end{array}$ \\
\hline Any-grade AEs & 16 & 2099 & 3100 & $0.66(0.58-0.74)$ & $<0.0001$ & 0.013 & 0.964 \\
PD-L1 inhibitor & 10 & 1414 & 2053 & $0.66(0.55-0.77)$ & $<0.0001$ & 0.060 & 0.474 \\
PD-1 inhibitor & 6 & 685 & 1047 & $0.66(0.60-0.72)$ & 0.007 & 0.910 & 0.452 \\
& & & & & & & \\
Grade $\geq 3$ AEs & 16 & 485 & 3100 & $0.13(0.09-0.18)$ & $<0.0001$ & 0.129 & 0.300 \\
PD-L1 inhibitor & 10 & 293 & 2053 & $0.11(0.05-0.17)$ & $<0.0001$ & 0.379 & 0.474 \\
PD-1 inhibitor & 6 & 192 & 1047 & $0.18(0.16-0.20)$ & 0.478 & 0.950 & 0.707 \\
\hline
\end{tabular}

Abbreviation: $\mathrm{AE}$ : adverse event.

To make a further analysis of the safety and efficacy of PD-1/PD-L1 inhibitors in treating advanced UC, we performed an up-to-date meta-analysis. In this updated meta-analysis, 18 studies comprising a total of 3, 144 patients diagnosed with advanced UC were included to explore the efficacy and safety of PD-1/ PD-L1 inhibitors in the treatment of these patients. Overall, the average ORR for PD-1/PD-L1 inhibitors was $0.20 \quad(95 \%$ CI $0.17-0.23$, Figure 2A). Furthermore, the pooled 1-year OS and 1-year PFS rates were 0.43 and 0.19 , respectively. Thus, PD-1/ PD-L1 immune checkpoint inhibitors elicited promising efficacy (Table 2). The underlying mechanism of action of PD-1/PD-L1 immune checkpoint inhibitors in the treatment of advanced UC could be the fact that PD-1/PD-L1 antibodies prevents the immune escape of tumor cells by blocking the binding of PD-1 on $\mathrm{T}$ cells to its ligand on tumor cells. The rates of any-grade and grade $\geq 3$ AEs were used to evaluate the drugs' safety profiles. The overall rate of any-grade AEs did not demonstrate a statistically significant difference in the PD-1 group compared with PD-L1 group. Noticeably, the pooled rates of grade $\geq 3$ AEs in the PD- 1 and PD-L1 groups were 0.18 and 0.11 , respectively. However, the related mechanism for this finding is unclear.

Substantial heterogeneity was detected in our metaanalysis due to different PD-L1 expression levels, PDL1/PD-1 inhibitors, studied drugs, visceral or lymph node-only metastasis, and either in the upper or lower tract. We conducted subgroup analyses to investigate the sources of the observed heterogeneity across studies. A large part of the detected heterogeneity may be explained by stratified analysis, which is based on differences in interventions across various studies, locations of metastases and primary tumors. Notwithstanding, we have confirmed an absence of significant publication bias in this meta-analysis either with the Begg's tests for each study. In addition, our sensitivity analyses revealed similar and robust results.
The research on PD-L1/PD-1 inhibitors has been receiving an increasing amount of attention over the recent years. In 2019, Zhang et al. published a metaanalysis consisting of clinical trials published until July 2019 [8]. The overall ORR was 0.20. However, 6 additional studies [17-19, 24, 26, 30] on this topic have been published between 2019 and 2020. Therefore, an updated meta-analysis was performed to ascertain the efficacy and safety of immune checkpoint inhibitors. Overall, the pooled ORR was 0.20 , and the efficacy and safety profiles were similar to those reported in the meta-analysis performed by Zhang et al. Nonetheless, among the subgroup analyses stratified by the location of metastasis or primary tumor, PD-1/PD-L1 inhibitors produced encouraging ORR in advanced UC patients with lymph node-only metastasis compared to those with visceral metastasis (0.41 VS. 0.17). Furthermore, patients with primary tumors situated in the lower tract had higher ORR compared to those with primary tumors in the upper tract (0.24 VS. 0.15). The findings observed from those two subgroups could have clinical guiding significance for the treatment of advanced UC by PD-1/PD-L1 inhibitors.

This study contains several important strengths that have been briefly mentioned below. This is an updated systematic epidemiologic assessment of the safety and efficacy of PD-1/PD-L1 inhibitors in treating advanced UC patients. Our summary analysis of 18 studies involving 3,144 patients with advanced UC provides a more stable association and reliable estimation. Furthermore, the findings observed in subgroup analyses grouped by location of metastasis or primary tumor have a promising benefit for the clinical management of those patients.

Withal, there are several potential limitations in our current study that need to be taken into account when interpreting the results. First and foremost are the limitations inherent to the majority of included studies, which were prone to have potential performance bias 
because most of them were different phase of clinical trials, and a larger number of RCTs on PD-1/PD-L1 inhibitors in treating advanced UC patients have not been conducted. Secondly, substantial heterogeneity was observed in this present analysis; although numerous subgroup analyses were conducted, the possible sources of heterogeneity were not identified. Thirdly, the included studies had various classifications of PD-L1 expression levels by different staining cut-off values, which might have an impact on the patient populations and mislead the true summary estimation. In summary, this updated meta-analysis not only confirmed the efficacy and safety of PD-1/PD-L1 inhibitors in treating advanced UC patients but also provided potential clinical significance for patients with lymph node-only metastases or primary tumors located in the lower tract. Nevertheless, further investigation mainly via RCTs is needed to confirm these findings.

\section{Availability of data and materials}

All data generated or analyzed during this study are included in the published articles.

\section{Abbreviations}

UC: Urothelial cancer; CI: Confidence interval; ORR: Objective response rate; OS: Overall survival; FS: Progression-free survival; PD-1: Programmed cell death 1; PD-L1: Programmed cell death-ligand 1; AEs: Adverse events; RCTs: Randomized control trials.

\section{AUTHOR CONTRIBUTIONS}

Yu Wang, Kunfeng Xie and Yunze Fang contributed to the data acquisition and data interpretation process. $\mathrm{Yu}$ Wang, Fei Li and Yuejun Du performed the statistical analyses and drafted the manuscript. Wanlong Tan and Lina Hou reviewed the results and provided help for paper writing. All the authors gave final approval of the submitted manuscript and reached an agreement to be equally responsible for every aspect of this work.

\section{CONFLICTS OF INTEREST}

The authors declare no conflicts of interest related to this study.

\section{FUNDING}

This study was supported by the Natural Science Foundation Committee of China (NSFC 81802941) (F.L.), the Natural Science Foundation of Guangdong Province of China (2018A0303130287) (F.L.), the Dean's research fund of Nanfang Hospital, the Southern Medical University (2020Z005) (F.L.), the Outstanding
Youth Development Scheme of Nanfang Hospital, Southern Medical University (2019J009) (F.L.), Guangdong Medical Research Fund (CZ2019114) (L.N.H), and the Dean's research fund of Nanfang Hospital, the Southern Medical University (2019B008) (L.N.H).

\section{Editorial note}

${ }^{\&}$ This corresponding author has a verified history of publications using a personal email address for correspondence.

\section{REFERENCES}

1. Cumberbatch MGK, Jubber I, Black PC, Esperto F, Figueroa JD, Kamat AM, Kiemeney L, Lotan Y, Pang K, Silverman DT, Znaor A, Catto JWF. Epidemiology of Bladder Cancer: A Systematic Review and Contemporary Update of Risk Factors in 2018. Eur Urol. 2018; 74:784-95.

https://doi.org/10.1016/j.eururo.2018.09.001 PMID:30268659

2. Witjes JA, Bruins HM, Cathomas R, Compérat EM, Cowan NC, Gakis G, Hernández V, Linares Espinós E, Lorch A, Neuzillet $Y$, Rouanne M, Thalmann GN, Veskimäe $\mathrm{E}$, et al. European Association of Urology Guidelines on Muscle-invasive and Metastatic Bladder Cancer: Summary of the 2020 Guidelines. Eur Urol. 2021; 79:82-104.

https://doi.org/10.1016/j.eururo.2020.03.055 PMID:32360052

3. Li F, Zhao H, Su M, Xie W, Fang Y, Du Y, Yu Z, Hou L, Tan W. HnRNP-F regulates EMT in bladder cancer by mediating the stabilization of Snail1 mRNA by binding to its 3' UTR. EBioMedicine. 2019; 45:208-19. https://doi.org/10.1016/j.ebiom.2019.06.017 PMID: $\underline{31221586}$

4. Zschäbitz S, Niegisch G. [Second-line treatment of metastatic urothelial carcinoma: Update immunooncology]. Urologe A. 2020; 59:804-09. https://doi.org/10.1007/s00120-020-01236-3 PMID:32472222

5. Zacharis A, Grüllich C. [First-line treatment of metastatic urothelial carcinoma: Update immunooncology]. Urologe A. 2020; 59:797-803. https://doi.org/10.1007/s00120-020-01235-4 PMID: $\underline{2500171}$

6. Kaplon H, Reichert JM. Antibodies to watch in 2019. MAbs. 2019; 11:219-38. https://doi.org/10.1080/19420862.2018.1556465 PMID:30516432

7. Fuhrmann C, Struck JP, Ivanyi P, Kramer MW, Hupe 
MC, Hensen B, Fürschke A, Peters I, Merseburger AS, Kuczyk MA, von Klot CJ. Checkpoint Inhibition for Metastatic Urothelial Carcinoma After Chemotherapy-Real-World Clinical Impressions and Comparative Review of the Literature. Front Oncol. 2020; 10:808.

https://doi.org/10.3389/fonc.2020.00808

PMID: $\underline{32528889}$

8. Zhang F, Liu Z, Liang J, Zhang F, Wu K, Zhou C, Lu Y, Wang $X$. The efficacy and safety of immunotherapy targeting the PD-1 pathway for advanced urothelial carcinoma: a meta-analysis of published clinical trials. Clin Transl Oncol. 2020; 22:1750-61. https://doi.org/10.1007/s12094-020-02316-8 PMID:32086783

9. Cochrane Handbook for Systematic Reviews of Interventions V5.2. 2017.

10. Li F, An SL, Zhou Y, Liang ZK, Jiao ZJ, Jing YM, Wan P, Shi XJ, Tan WL. Milk and dairy consumption and risk of bladder cancer: a meta-analysis. Urology. 2011; 78:1298-305.

https://doi.org/10.1016/j.urology.2011.09.002 PMID:22137695

11. Li Y, Yang D, Chen P, Yin X, Sun J, Li H, Ren G. Efficacy and safety of neoadjuvant chemotherapy regimens for triple-negative breast cancer: a network metaanalysis. Aging (Albany NY). 2019; 11:6286-311.

https://doi.org/10.18632/aging.102188 PMID:31446432

12. Li F, Zhou Y, Hu RT, Hou LN, Du YJ, Zhang XJ, Olkkonen VM, Tan WL. Egg consumption and risk of bladder cancer: a meta-analysis. Nutr Cancer. 2013; 65:538-46.

https://doi.org/10.1080/01635581.2013.770041 PMID:23659445

13. Petrylak DP, Powles T, Bellmunt J, Braiteh F, Loriot $\mathrm{Y}$, Morales-Barrera R, Burris HA, Kim JW, Ding B, Kaiser C, Fassò M, O'Hear C, Vogelzang NJ. Atezolizumab (MPDL3280A) Monotherapy for Patients With Metastatic Urothelial Cancer: Long-term Outcomes From a Phase 1 Study. JAMA Oncol. 2018; 4:537-44. https://doi.org/10.1001/jamaoncol.2017.5440 PMID:29423515

14. Rosenberg JE, Hoffman-Censits J, Powles $T$, van der Heijden MS, Balar AV, Necchi A, Dawson N, O'Donnell $\mathrm{PH}$, Balmanoukian A, Loriot $\mathrm{Y}$, Srinivas $\mathrm{S}$, Retz MM, Grivas $P$, et al. Atezolizumab in patients with locally advanced and metastatic urothelial carcinoma who have progressed following treatment with platinumbased chemotherapy: a single-arm, multicentre, phase 2 trial. Lancet. 2016; 387:1909-20.

https://doi.org/10.1016/S0140-6736(16)00561-4 PMID:26952546
15. Pal SK, Hoffman-Censits J, Zheng H, Kaiser C, Tayama $\mathrm{D}$, Bellmunt J. Atezolizumab in Platinum-treated Locally Advanced or Metastatic Urothelial Carcinoma: Clinical Experience from an Expanded Access Study in the United States. Eur Urol. 2018; 73:800-06.

https://doi.org/10.1016/i.eururo.2018.02.010 PMID:29478735

16. Powles T, Durán I, van der Heijden MS, Loriot $Y$, Vogelzang NJ, De Giorgi U, Oudard S, Retz MM, Castellano D, Bamias A, Fléchon A, Gravis G, Hussain $S$, et al. Atezolizumab versus chemotherapy in patients with platinum-treated locally advanced or metastatic urothelial carcinoma (IMvigor211): a multicentre, open-label, phase 3 randomised controlled trial. Lancet. 2018; 391:748-57.

https://doi.org/10.1016/S0140-6736(17)33297-X PMID:29268948

17. Galsky MD, Arija JÁA, Bamias A, Davis ID, De Santis M, Kikuchi E, Garcia-Del-Muro X, De Giorgi U, Mencinger $M$, Izumi K, Panni S, Gumus $M$, Özgüroğlu $M$, et al, and IMvigor130 Study Group. Atezolizumab with or without chemotherapy in metastatic urothelial cancer (IMvigor130): a multicentre, randomised, placebocontrolled phase 3 trial. Lancet. 2020; 395:1547-57. https://doi.org/10.1016/S0140-6736(20)30230-0 PMID: $\underline{32416780}$

18. Vander Velde $N$, Guerin A, lonescu-Ittu R, Shi S, Wu EQ, Lin SW, Hsu LI, Saum KU, de Ducla S, Wang J, Li S, Thåström A, Liu S, et al. Comparative Effectiveness of Non-cisplatin First-line Therapies for Metastatic Urothelial Carcinoma: Phase 2 IMvigor210 Study Versus US Patients Treated in the Veterans Health Administration. Eur Urol Oncol. 2019; 2:12-20. https://doi.org/10.1016/j.euo.2018.07.003 PMID:30929841

19. Powles T, Eder JP, Fine GD, Braiteh FS, Loriot $Y$, Cruz C, Bellmunt J, Burris HA, Petrylak DP, Teng SL, Shen X, Boyd Z, Hegde PS, et al. MPDL3280A (anti-PD-L1) treatment leads to clinical activity in metastatic bladder cancer. Nature. 2014; 515:558-62.

https://doi.org/10.1038/nature13904 PMID:25428503

20. Powles T, O'Donnell PH, Massard C, Arkenau HT, Friedlander TW, Hoimes CJ, Lee JL, Ong M, Sridhar SS, Vogelzang NJ, Fishman MN, Zhang J, Srinivas S, et al. Efficacy and Safety of Durvalumab in Locally Advanced or Metastatic Urothelial Carcinoma: Updated Results From a Phase 1/2 Open-label Study. JAMA Oncol. 2017; 3:e172411.

https://doi.org/10.1001/jamaoncol.2017.2411 PMID:28817753

21. Massard C, Gordon MS, Sharma S, Rafii S, Wainberg ZA, Luke J, Curiel TJ, Colon-Otero G, Hamid O, Sanborn RE, 
O'Donnell PH, Drakaki A, Tan W, et al. Safety and Efficacy of Durvalumab (MEDI4736), an AntiProgrammed Cell Death Ligand-1 Immune Checkpoint Inhibitor, in Patients With Advanced Urothelial Bladder Cancer. J Clin Oncol. 2016; 34:3119-25.

https://doi.org/10.1200/JCO.2016.67.9761 PMID:27269937

22. Patel MR, Ellerton J, Infante JR, Agrawal M, Gordon $M$, Aljumaily R, Britten CD, Dirix L, Lee KW, Taylor M, Schöffski P, Wang D, Ravaud A, et al. Avelumab in metastatic urothelial carcinoma after platinum failure (JAVELIN Solid Tumor): pooled results from two expansion cohorts of an open-label, phase 1 trial. Lancet Oncol. 2018; 19:51-64.

https://doi.org/10.1016/S1470-2045(17)30900-2 PMID:29217288

23. Apolo $A B$, Infante JR, Balmanoukian $A$, Patel $M R$, Wang D, Kelly K, Mega AE, Britten CD, Ravaud A, Mita $A C$, Safran $H$, Stinchcombe TE, Srdanov $M$, et al. Avelumab, an Anti-Programmed Death-Ligand 1 Antibody, In Patients With Refractory Metastatic Urothelial Carcinoma: Results From a Multicenter, Phase Ib Study. J Clin Oncol. 2017; 35:2117-24.

https://doi.org/10.1200/JCO.2016.71.6795 PMID:28375787

24. Vuky J, Balar AV, Castellano D, O'Donnell PH, Grivas P, Bellmunt J, Powles T, Bajorin D, Hahn NM, Savage MJ, Fang X, Godwin JL, Frenkl TL, et al. Long-Term Outcomes in KEYNOTE-052: Phase II Study Investigating First-Line Pembrolizumab in CisplatinIneligible Patients With Locally Advanced or Metastatic Urothelial Cancer. J Clin Oncol. 2020; 38:2658-66.

https://doi.org/10.1200/JCO.19.01213

PMID:32552471

25. Bellmunt J, de Wit R, Vaughn DJ, Fradet $Y$, Lee JL, Fong L, Vogelzang NJ, Climent MA, Petrylak DP, Choueiri TK, Necchi A, Gerritsen W, Gurney H, et al, and KEYNOTE-045 Investigators. Pembrolizumab as Second-Line Therapy for Advanced Urothelial Carcinoma. N Engl J Med. 2017; 376:1015-26.

https://doi.org/10.1056/NEJMoa1613683 PMID:28212060

26. Nishiyama $H$, Yamamoto $Y$, Sassa $N$, Nishimura $K$, Fujimoto K, Fukasawa S, Yokoyama M, Enokida $H$, Takahashi K, Tanaka Y, Imai K, Shimamoto T, Perini R, et al. Pembrolizumab versus chemotherapy in recurrent, advanced urothelial cancer in Japanese patients: a subgroup analysis of the phase 3 KEYNOTE-045 trial. Int J Clin Oncol. 2020; 25:165-74. https://doi.org/10.1007/s10147-019-01545-4 PMID: $\underline{31729625}$

27. Plimack ER, Bellmunt J, Gupta S, Berger R, Chow LQ,
Juco J, Lunceford J, Saraf S, Perini RF, O'Donnell PH. Safety and activity of pembrolizumab in patients with locally advanced or metastatic urothelial cancer (KEYNOTE-012): a non-randomised, open-label, phase 1b study. Lancet Oncol. 2017; 18:212-20.

https://doi.org/10.1016/S1470-2045(17)30007-4 PMID:28081914

28. Sharma $P$, Retz $M$, Siefker-Radtke $A$, Baron $A$, Necchi A, Bedke J, Plimack ER, Vaena D, Grimm MO, Bracarda $S$, Arranz JÁ, Pal S, Ohyama C, et al. Nivolumab in metastatic urothelial carcinoma after platinum therapy (CheckMate 275): a multicentre, single-arm, phase 2 trial. Lancet Oncol. 2017; 18:312-22. https://doi.org/10.1016/S1470-2045(17)30065-7 PMID:28131785

29. Sharma P, Callahan MK, Bono P, Kim J, Spiliopoulou P, Calvo E, Pillai RN, Ott PA, de Braud F, Morse M, Le DT, Jaeger D, Chan E, et al. Nivolumab monotherapy in recurrent metastatic urothelial carcinoma (CheckMate 032): a multicentre, open-label, two-stage, multi-arm, phase 1/2 trial. Lancet Oncol. 2016; 17:1590-98. https://doi.org/10.1016/S1470-2045(16)30496-X PMID:27733243

30. Shen L, Guo J, Zhang $Q$, Pan H, Yuan $Y$, Bai $Y$, Liu T, Zhou Q, Zhao J, Shu Y, Huang X, Wang S, Wang J, et al. Tislelizumab in Chinese patients with advanced solid tumors: an open-label, non-comparative, phase $1 / 2$ study. J Immunother Cancer. 2020; 8:e000437. https://doi.org/10.1136/jitc-2019-000437 PMID: $\underline{32561638}$

31. Todenhöfer T, Boegemann M. [Immunotherapy in bladder cancer-quo vadis? Update on current trials and developments]. Urologe A. 2020; 59:810-16. https://doi.org/10.1007/s00120-020-01237-2 PMID:32468092

32. Galsky MD, Chen GJ, Oh WK, Bellmunt J, Roth BJ, Petrioli R, Dogliotti L, Dreicer R, Sonpavde G. Comparative effectiveness of cisplatin-based and carboplatin-based chemotherapy for treatment of advanced urothelial carcinoma. Ann Oncol. 2012; 23:406-10.

https://doi.org/10.1093/annonc/mdr156 PMID:21543626

33. Galsky MD, Hahn NM, Rosenberg J, Sonpavde G, Hutson T, Oh WK, Dreicer R, Vogelzang N, Sternberg $\mathrm{CN}$, Bajorin DF, Bellmunt J. Treatment of patients with metastatic urothelial cancer "unfit" for Cisplatinbased chemotherapy. J Clin Oncol. 2011; 29:2432-38. https://doi.org/10.1200/JCO.2011.34.8433 PMID:21555688

34. Bellmunt J, Powles T, Vogelzang NJ. A review on the evolution of PD-1/PD-L1 immunotherapy for bladder cancer: The future is now. Cancer Treat Rev. 2017; 54:58-67. 
https://doi.org/10.1016/j.ctrv.2017.01.007

PMID:28214651 